\title{
Análise da morfometria nuclear: descrição da metodologia e o papel dos softwares de edição de imagem
}

\section{Nuclear morphometric analysis: description of the methodology and the role of image-editing softwares}

Carlos Eduardo de Andrea'; Luiz Fernando Bleggi-Torres'; Maria Teresa de Seixas Alves ${ }^{3}$

\section{unitermos \\ Análise de imagens \\ Morfometria}

Morfometria nuclear

\section{resumo}

A análise morfométrica de imagens é utilizada em patologia para se obter dados precisos de componentes celulares e teciduais, por meio da mensuração e contagem, que são importantes para o diagnóstico e prognóstico de vários tumores. Essa técnica, mesmo sendo ferramenta útil e de baixo custo, mantém-se restrita a poucos centros de diagnóstico ou pesquisa. O objetivo deste trabalho é apresentar e descrever o método de morfometria nuclear com a utilização complementar de softwares de edição de imagens, fazendo a análise detalhada das etapas críticas do processo. A correção de problemas encontrados durante a captura das imagens, como temperatura e matiz da cor erroneamente ajustados, e de imagens com iluminação desigual provenientes de microscópio mal regulado, é fundamental para a correta análise. Assim, utilizando o recurso máscara de cor disponível em vários softwares de editoração de imagens, é possível selecionar e separar objetos de cores semelhantes, resultando numa imagem final que apresenta somente os objetos de interesse para análise. A morfometria é realizada por meio de softwares específicos como o ImageTool (versão 3.0), que possibilitam a mensuração automática de um ou mais parâmetros, com precisão e em curto intervalo de tempo. A interface do ImageTool permite ainda trabalhar simultaneamente com a imagem original e a imagem processada em que se fez a seleção das cores semelhantes. Dessa forma, pode-se confrontar o objeto que está sendo selecionado com o mesmo objeto na sua imagem original, garantindo assim correta seleção. Esse sistema possibilita conferência interobservador de $100 \%$ dos objetos, o que permite desprezar aqueles que posteriormente constatou-se que não deveriam ter sido selecionados e mensurados.

\footnotetext{
The morphometric analysis of images, which comprises measuring and counting, is used in pathology to obtain accurate data of cellular and tissue components that are important for diagnosis and prognosis of several tumors. Although it is a useful and low-cost tool, it is restricted to a few diagnosis and research centers. The objective of this study is to present and describe the method of nuclear morphometry with supplementary use of image-editing softwares, making detailed analysis of the critical stages of the process. The correction of problems found during the capture of the image, such as erroneously adjusted color temperature and color shade and unevenly illuminated images from an improperly adjusted microscope, is fundamental for the accurate analysis. Through the color mask resource present in countless image-editing softwares, it is possible to select and to separate objects of similar colors, resulting in a final image that shows only the objects of interest for analysis. Morphometry is accomplished through a specific software, such as ImageTool (version 3.00), that permits the accurate automatic measuring of one or more parameters in a short interval of time. The interface of ImageTool allows one to work simultaneously with the original and the processed images, in which the similar colors were selected. Therefore, it is possible to confront the selected object with the same object in its original image, what ensures its exact selection. This system allows interobserver comparison of $100 \%$ of the objects, enabling the rejection of those that should not have been selected and measured.
}

abstract

key words

Image analysis

Morphometry

Nuclear morphometry 


\section{Introdução}

A análise de imagem é utilizada em patologia para se obter dados precisos, mediante mensuração e contagem. As mensurações de imagens microscópicas são tão antigas quanto o próprio microscópio. Antonie van Leeuwenhoek, no século XVII, desenvolveu um método para mensurar objetos microscópicos, tendo como referência grãos de areia e fios de cabelos de diferentes tamanhos ${ }^{(19)}$. Em 1674, utilizando essa metodologia, ele descobriu que o eritrócito humano era 25 mil vezes menor do que um grão de areia pequeno, correspondendo a $8,5 \mu \mathrm{m}^{(19)}$.

Informações importantes com implicações diagnósticas e prognósticas podem ser obtidas por meio da avaliação morfométrica de células e componentes teciduais.

A morfometria nuclear estuda alguns parâmetros do núcleo como tamanho, forma e padrão cromatínico. Essas características nas células malignas são conhecidamente diferentes daquelas observadas nas não-malignas. Com base nessas diferenças, vários autores propuseram que a morfometria nuclear seja utilizada para aprimorar os parâmetros diagnósticos e fornecer fatores prognósticos confiáveis $(2,7,8,11,21,29)$.

Tem sido descrita significativas alterações de parâmetros morfométricos nucleares em células localizadas na periferia de neoplasias malignas ${ }^{(10,14)}$. Essas observações sugerem a hipótese de que essas alterações morfológicas ocorram nas células antes da manifestação clínica do tumor. Portanto a morfometria nuclear poderia auxiliar como biomarcador para avaliar essas alterações displásicas e o seu potencial carcinogênico 3 (3, 24, 26).

Em 1982, Diamond et al. ${ }^{(9)}$ utilizaram pela primeira vez a medição do tamanho nuclear como fator prognóstico em adenocarcinomas de próstata. Outros autores realizaram o mesmo experimento em outros órgãos, e confirmaram que as metástases são mais freqüentemente observadas em neoplasias cujos núcleos são grandes. Em contraste, neoplasia com núcleos pequenos apresentam melhor prognóstico ${ }^{(9,15,27)}$.

Apel et al. ${ }^{(1)}$ estudaram morfometricamente os núcleos de 16 casos de osteossarcoma (OS) central de alto grau, que foram divididos em dois grupos (respondedores e não-respondedores) com base no grau de necrose à quimioterapia pré-operatória. As células do OS nos 10 casos respondedores apresentaram marcado pleomorfismo nuclear e aumento de tamanho, tanto celular como nuclear, e esses eram ainda hipercromáticos, únicos ou múltiplos.

O valor médio da área desses núcleos foi $66 \pm 41 \mu \mathrm{m}^{2}$. As células tumorais não-respondedoras apresentaram pouco pleomorfismo nuclear, com núcleos arredondados, ovóides ou fusiformes, e valor médio da área nuclear de $38 \pm 18 \mu \mathrm{m}^{2}$. Assim, concluiu que o tamanho do núcleo foi capaz de diferenciar os casos de osteossarcomas respondedores dos não-respondedores à quimioterapia pré-operatória( ${ }^{(1)}$.

Ishida et al.(16) estudaram a correlação entre o grau histológico de 24 casos de tumores cartilaginosos e sua morfometria nuclear. A média da área nuclear nas lesões condróides benignas (sete casos) foi $15,71 \pm 1,98 \mu \mathrm{m}^{2}$, enquanto nos condrossarcomas bem-diferenciados foi 23,01 $\pm 2,3 \mu \mathrm{m}^{2}$, sendo a média para os condrossarcomas de alto grau de $28,59 \pm 6,78 \mu \mathrm{m}^{2}$. Eles também demonstraram que houve diferença significativa no que se refere ao tamanho da área nuclear de lesões cartilaginosas benignas e malignas.

Nativ et al.(22), em 1995, avaliando carcinomas renais não-metastáticos, observaram que a forma nuclear era, isoladamente, o fator mais importante para predizer a recorrência da neoplasia; no entanto os tumores compostos por núcleos mais elípticos apresentavam pior prognóstico. Outros estudos demonstraram que os carcinomas de células renais com núcleos grandes $\left(>35 \mu^{2}\right)$ têm tempo médio de sobrevida significativamente menor ${ }^{(12,13)}$.

Breslow et al.(5), em 1999, concluíram que a morfometria nuclear não prediz o tempo de sobrevida livre de doença para pacientes com tumor de Wilms. Entretanto menciona que a morfometria é técnica potencialmente útil para fornecer informações objetivas, predizendo a resposta ao tratamento de pacientes com câncer.

Em 2001, Milord et al. ${ }^{(20)}$ utilizaram a morfometria nuclear como parâmetro objetivo para diferenciar o carcinoma in situ de bexiga de outras lesões uroteliais planas. Eles observaram que o tamanho do núcleo das células uroteliais normais era aproximadamente duas vezes o do linfócito e que nas lesões planas de carcinoma in situ era cinco vezes o tamanho do linfócito.

Parâmetros morfométricos também foram utilizados no estudo de neoplasias ósseas e de partes moles, como nos trabalhos de Oda et al.(23), em 1995, avaliando tumor neuroectodérmico primitivo (PNET)/sarcomas de Ewing ósseos, e de Kazanowska et al.(17), em 2004, que estudaram rabdomiossarcomas humanos.

Kazanowska et al. ${ }^{(17)}$ concluíram que a morfometria nuclear pode auxiliar na subclassificação desses tumores, e que parâmetros como coeficiente de arredondamento (CA) nuclear e tendência do núcleo a ser elíptico podem ser utilizados como fatores prognósticos. Pacientes com $\mathrm{CA}<0,6$ e com mais de $10 \%$ de células fusiformes tiveram melhor prognóstico por 
apresentarem, na visão dos autores, células mais bem diferenciadas, tendendo a ser tumores menos agressivos. Já aqueles com núcleos pequenos e com mais de $10 \%$ das células com núcleos redondos tendiam a ter pior prognóstico, talvez por serem mais anaplásicos ${ }^{(17)}$.

Há mais de 30 anos se discute na literatura a utilização da análise morfométrica quantitativa como ferramenta útil e de baixo custo, entretanto o emprego dessa técnica mantém-se restrito a poucos centros de diagnóstico ou de pesquisa. Uma das prováveis razões é que a literatura apresenta limitadas evidências de que essa técnica possa realmente prover algoritmos estáveis, específicos, seletivos e reprodutíveis, que possam ser utilizados no diagnóstico, prognóstico e avaliação das neoplasias ${ }^{(3,}$ $18,25,26)$.

Essa situação parece que se agravou ainda mais devido ao aparecimento de softwares que fornecem grande número de informações morfométricas derivadas da mesma base de dados de imagem. É possível questionar a utilidade desses novos dados e se tais mensurações representam achados adicionais ou são puramente desdobramentos de parâmetros preestabelecidos. Portanto, a redução do número de parâmetros deve ser prioridade para tornar a análise morfométrica uma técnica mais viável, acessível e atrativa.

Este trabalho tem por objetivo apresentar e descrever o método de morfometria nuclear com a utilização complementar de softwares de edição de imagens, fazendo análise detalhada das etapas críticas do processo.

\section{Análise morfométrica}

Morfometria é descrição quantitativa dos achados geométricos de estruturas de qualquer dimensão $0^{(6,19)}$. Isso inclui a planimetria, que é o processo de medição horizontal, ou seja, é a medida das áreas das superfícies planas, e a estereometria que está relacionada à medida dos sólidos ${ }^{(19)}$. A Tabela mostra diversos parâmetros que podem ser obtidos por meio da planimetria.

O olho humano é sensível a muitas variáveis, como luminosidade, diferenças de contraste e brilho, que poderão resultar em interpretações e conclusões subjetivas e específicas do analisador. Portanto, um dos objetivos da morfometria é eliminar a subjetividade e permitir maior reprodutibilidade interobservador. Dessa forma, vários autores recomendam a análise de pelo menos cinco campos microscópicos em aumento de 400 vezes ou 1.000 vezes $(9,17,20,23,27)$. De rotina, utilizamos a análise de 10 campos de grande aumento e/ou a avaliação de pelo menos 100 núcleos por caso.

Em nosso serviço, a análise morfométrica de imagens é realizada de acordo com o seguinte protocolo, considerando com detalhes as etapas críticas do processo:

\section{Captura das imagens}

A qualidade das imagens estudadas pode ser comprometida por diversos fatores, como a preparação das amostras (fixação, espessura do corte, coloração etc.), o equipamento utilizado (qualidade do microscópio, tipo e resolução da câmera digital etc.), bem como as condições

\section{Tabela Principais parâmetros fornecidos pela análise morfométrica}

\begin{tabular}{|c|c|}
\hline Parâmetro & Descrição \\
\hline Área & Medida por meio do número de pixels no polígono \\
\hline Perímetro & Comprimento do limite externo do polígono. \\
\hline Coeficiente de arredondamento & $\begin{array}{l}\text { Fornece um valor entre } 0 \text { e } 1 \text {; quanto maior o valor, mais redondo é o objeto. Se } \\
\text { a relação for igual a } 1,0 \text { objeto é um círculo perfeito }\end{array}$ \\
\hline Alongamento & $\begin{array}{l}\text { Relação do comprimento do eixo principal com o comprimento do eixo } \\
\text { secundário. } 0 \text { resultado é um valor entre } 0 \text { e } 1 \text {. Se } 0 \text { alongamento for } 1 \text {, o objeto } \\
\text { é aproximadamente circular ou quadrado; contudo, se o resultado for }<1,0 \\
\text { objeto se torna mais alongado }\end{array}$ \\
\hline Comprimento do eixo principal & Comprimento da linha mais longa que pode ser desenhada pelo objeto \\
\hline Ângulo do eixo principal & Ângulo entre os eixos horizontal e principal, em graus \\
\hline Comprimento do eixo secundário & $\begin{array}{l}\text { Comprimento da linha mais longa que pode ter o objeto perpendicular ao eixo } \\
\text { principal }\end{array}$ \\
\hline Ângulo de eixo secundário & Ângulo entre o eixo horizontal e o eixo secundário, em graus. \\
\hline
\end{tabular}


de captura da imagem (tipo e quantidade de iluminação, cor, foco etc. $)^{(30)}$.

O sistema de captura de imagem que utilizamos corresponde ao microscópio Olympus BX40, acoplado à câmara digital Sony CCD-IRIS, que está conectada ao computador Dell (Dimension 4400), com microprocessador Pentium 4 (2.0 GHz), $1 \mathrm{~GB}$ RAM e placa digitalizadora de imagens, trabalhando em ambiente Windows (XP Professional).

Em geral, os principais problemas encontrados durante a captura das imagens são "temperatura" e matiz da cor erroneamente ajustados e imagens com iluminação desigual proveniente de microscópio mal regulado.

É bom relembrar que toda imagem capturada é resultado da combinação do objeto que está sendo fotografado com o seu fundo (Figura 1). Para melhorar a qualidade da imagem é fundamental ter a luz do microscópio bem alinhada, o que pode ser obtido utilizando o princípio de iluminação de Köehler. Problemas com o fundo da imagem capturada, como tender para determinada cor e/ou o fato de a luz estar heterogeneamente distribuída, poderão dificultar a seleção (automática ou semi-automática) pelos softwares de análise de imagens dos objetos de interesse.

\section{Seleção automática dos pixels}

O recurso "máscara de cor" disponível em vários softwares de editoração de imagens, como o Corel Photo Paint 11, permite selecionar pixels de cor específicos e, automaticamente, o software selecionará também pixels semelhantes (Figura 2).

Pixel é o nome atribuído a picture element (elemento de imagem) e corresponde à menor área retangular da imagem. Cada pixel é formado por cores diferentes e, me-

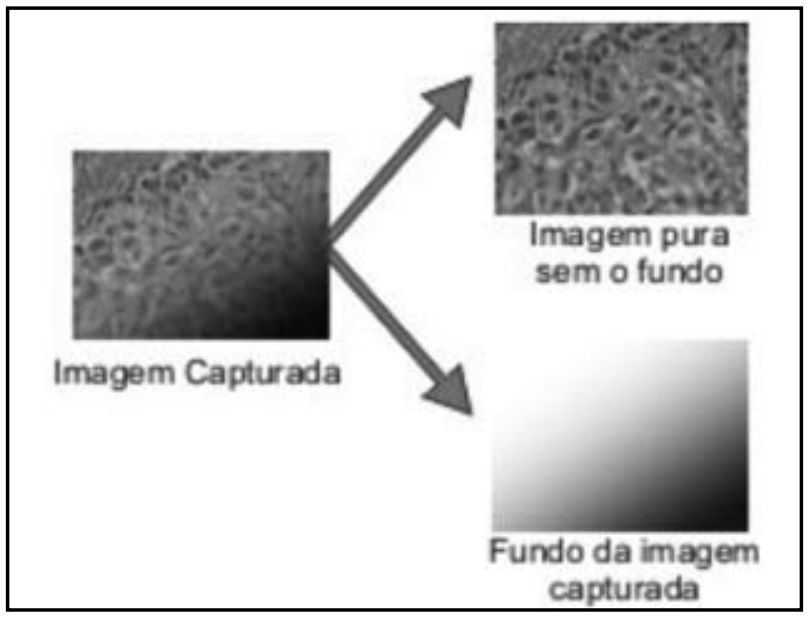

Figura 1 - Imagem capturada = objeto que está sendo fotografado + fundo. A correção das irregularidades do fundo da imagem é fundamental para a correta análise morfométrica

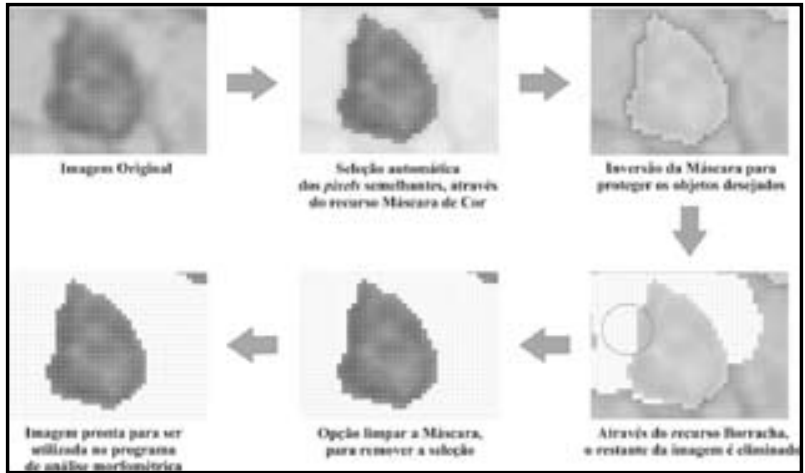

Figura 2 - Método adotado para isolar os objetos de pixels semelhantes utilizando 0 software Corel Photo Paint 11

diante a combinação dessas cores, é possível representar qualquer cor.

Quando se deseja separar somente os núcleos presentes na imagem, deve-se, utilizando o recurso "máscara de cor", selecionar a tonalidade do pixel que mais predomina no núcleo e, automaticamente, o programa separará esses pixels e também aqueles com tons semelhantes.

Em seguida, por meio da opção inversão da máscara, deve-se proteger os objetos desejados e, dessa forma, o restante da imagem poderá ser apagado pela ferramenta borracha, ficando apenas os objetos que serão analisados morfometricamente.

Com essa técnica conseguimos selecionar facilmente 0 maior número de objetos, garantindo eficiência e precisão. O resultado final desse processo poderá ser salvo como arquivo de imagens ou, pelo recurso copiar/colar, poderá ser transferido para o software de análise de imagens.

O sucesso para obter adequada individualização das cores está relacionado à seleção correta da cor com auxílio do recurso "máscara de cor". Assim, utilizamos o critério de selecionar os tons mais fortes da cor, uma vez que o programa automaticamente selecionará todas as tonalidades semelhantes.

A correção da iluminação é fundamental para a correta análise morfométrica, pois imagens homogeneamente iluminadas não apresentarão diferenças nas tonalidades das cores, o que possibilitará que todos os objetos com tons de cor semelhantes sejam selecionados pelo software.

Inicialmente, cerca de $80 \%$ a $90 \%$ dos pixels semeIhantes são selecionados. Em seguida, por meio de ajuste fino, que consiste em adicionar ou retirar cores utilizando o recurso "máscara de cor", obtém-se excelente separação e individualização dos objetos de interesse. O resultado final poderá ser então utilizado nos softwares de análise de imagens. 
Há vários programas disponíveis no mercado com o recurso "máscara de cor", como o GIMP (GNU Image Manipulation Program) e o Adobe Photoshop.

\section{Software de análise de imagem}

O uso de técnicas de análise de imagens em anatomia patológica é cada vez mais freqüente, e combina a utilização de sistema de captura de imagens com softwares específicos. Atualmente, há vários softwares disponíveis no mercado, alguns deles gratuitos, como o Image Tool (versão 3.0) para Windows fornecido pelo Health Science Center (HSC) da Universidade do Texas (EUA) ${ }^{(28)}$.

Esses softwares possibilitam, em curto intervalo de tempo, a mensuração automática de um ou mais parâmetros. Os principais parâmetros analisados estão representados na Tabela. Outros parâmetros podem ser ainda analisados, como o diâmetro de Feret (DF), densidade, níveis de cinza presentes na imagem, entre outros.

O CA pode ser obtido pela fórmula:

\section{$\mathrm{CA}=4 \pi \times$ área}

perímetro ${ }^{2}$

A análise morfométrica e a seleção dos objetos nos softwares de análise de imagens podem ser realizadas de forma manual, semi-automática ou automática. Além disso, a seleção pode ser feita de forma combinada, em que os objetos são manualmente selecionados como, por exemplo, somente os núcleos íntegros, sem sobreposição, e que não estão em apoptose ou mitose, e o programa de computador realiza automaticamente a mensuração dos objetos desejados (Figura 3).

O software Image Tool trabalha em escala de cinza e, por essa razão, apresenta a opção para converter a imagem co-

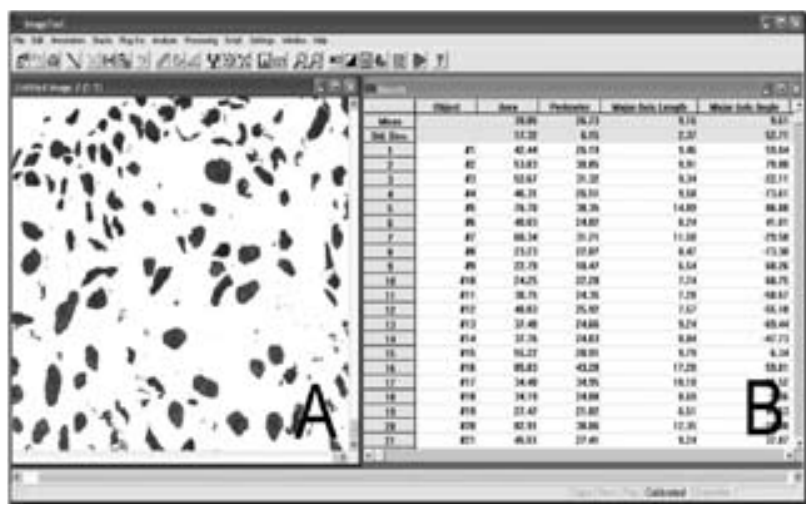

Figura 3 - Interface do programa Image Tool versão 3.0 para Windows. A: os objetos selecionados são circundados e numerados em vermelho; $B$ : os resultados numerados obtidos a partir da seleção anterior lorida ou RGB em escala de cinza (color to gray). Entretanto, após essa conversão, várias informações valiosas fornecidas pela presença das cores serão perdidas, como, por exemplo, as inúmeras tonalidades que a coloração nuclear pode assumir não serão mais distinguidas. Conseqüentemente, o software de análise de imagens pode confundir o núcleo com outras estruturas celulares. Portanto, a utilização de softwares complementares, que permitem a seleção precisa dos pixels desejados, tende a diminuir essas perdas, conforme descrito anteriormente.

A interface do software Image Tool 3.0 permite abrir, ao mesmo tempo, a imagem original e a imagem processada, ou seja, aquela em que se fez a seleção dos pixels semeIhantes, deixando, por exemplo, os núcleos individualizados, o que possibilita confrontar o objeto que está sendo selecionado para mensuração com o mesmo objeto na imagem original, garantindo assim uma correta e precisa seleção (Figura 4).

Os objetos selecionados são numerados e circundados em vermelho. Cada número corresponde a um objeto selecionado e também aos resultados obtidos pela sua mensuração (Figura 3). Isso possibilita conferência interobservador de $100 \%$ dos objetos mensurados, desprezando assim alguns que posteriormente chegou-se a conclusão de que não deveriam ter sido selecionados e mensurados.

Utilizamos o modo interativo, em que os objetos são selecionados manualmente e o programa de computador realiza automaticamente a mensuração dos objetos desejados.

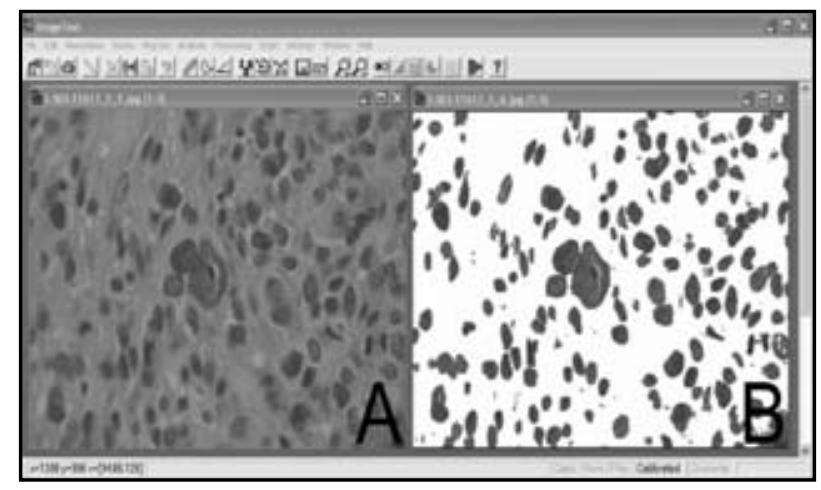

Figura 4 - Interface do programa Image Tool versão 3.0 para Windows. A: imagem original; $B$ : após a seleção dos pixels, os núcleos estão individualizados. Cada núcleo íntegro é selecionado e a mensuração é realizada naqueles que estão circundados em vermelhos

\section{Conclusão e perspectivas}

Informações importantes com implicações diagnósticas e prognósticas podem ser obtidas pela análise morfométrica. A metodologia apresentada e descrita neste 
trabalho tem-se mostrado ferramenta útil e de baixo custo, podendo ser realizada em coloração padrão de hematoxilina-eosina (HE).

Outra vantagem é a possibilidade de confrontar a imagem original com a processada, na qual os objetos de interesse foram individualizados, garantindo maior acurácia na seleção dos objetos, uma vez que todos os elementos indesejados na imagem foram excluídos. Além disso, a conferência interobservador pode ser feita em $100 \%$ dos objetos mensurados, permitindo futura exclu- são daqueles que não deveriam ter sido selecionados e mensurados.

A metodologia que estamos utilizando para análise morfométrica, especialmente a nuclear, tem-se provado factível de ser empregada, podendo se constituir numa ferramenta auxiliar para a obtenção de determinados parâmetros que poderão ajudar na subclassificação histológica de alguns tumores e/ou ser fator prognóstico em outros, além de contribuir para a pesquisa nas mais diversas áreas de abrangência da anatomia patológica.

\section{Referências}

1. APEL, R. et al. Nuclear polymorphism in osteosarcomas as a prognostic factor for the effect of chemotherapy: a quantitative study. Virchows Arch A Pathol Anat Histopathol, v. 405, n. 2, p. 215-23, 1985.

2. BAAK, J.P. The principles and advances of quantitative pathology. Anal Quant Cytol Histol, v. 9, p. 89-95, 1987.

3. BACUS, J.W. et al. Quantitation of preinvasive neoplastic progression in animal models of chemical carcinogenesis. J Cell Biochem Suppl, v. 29, p. 21-38, 1997.

4. BOONE, C.W. et al. Quantitative grading of rat esophageal carcinogenesis using computer-assisted image tile analysis. Cancer Epidemiol Biomarkers \& Prev, v. 9 , p. 495-500, 2000.

5. BRESLOW, N.E. et al. Nuclear morphometry and prognosis in favorable histology Wilms' tumor: a prospective reevaluation. J Clin Oncology, v. 17, n. 7, p. 2123-6, 1999.

6. BUHMEIDA, A. Quantitative pathology: historical background, clinical research and application of nuclear morphometry and DNA image cytometry. Libyan J Med, AOP: 060911, 2006.

7. CARR, I.; PETTIGREW, N. How malignant is malignant? A brief review of the microscopic assessment of human neoplasms, and the prediction of whether they will metastasize and kill. Clin Exp Metastasis, v. 9, p. 12737, 1991.

8. COLLAN, Y. et al. Application of morphometry in tumor pathology. Anal Quant Cytol Histol, v. 9, p. 79-88, 1987.

9. DIAMOND, D.A. et al. A new method to assess metastatic potential of human prostate cancer: relative nuclear roundness. J. Urol., v. 128, p. 729-34, 1982.

10. DOUDKINE, A. et al. Nuclear texture measurements in image cytometry. Pathologica, v. 87, p. 286-99, 1995.

11. GIL, J. et al. Image analysis and morphometry in the diagnosis of breast cancer. Microsc Res Tech, v. 59. p. 109-18, 2002.

12. GILCHRIST, K.W. et al. Prognostic significance of nuclear sizing in renal cell carcinoma. Urology, v. 24, p. 1224, 1981.

13. GUTIERREZ, J.L. et al. Nuclear morphometry in prognosis of renal adenocarcinoma. Urology, v. 39, p. 130-4, 1992.
14. HAMILTON, P.W. et al. Nuclear texture measurements in normal colorectal glands. Anal Quant Cytol Histol, v. 17, p. 397-405, 1995.

15. HOGBERG, T. et al. Nuclear morphometry: a strong prognostic factor for survival after secondary surgery in advanced ovarian cancer. Int J Gynecol Cancer, v. 2, p. 198-206, 1992.

16. ISHIDA, T. et al. Histological grading and morphometric analysis of cartilaginous tumours. Virchows Arch A Pathol Anat Histopathol. v. 418, n. 2, p. 149-55, 1991.

17. KAZANOWSKA, B. et al. The role of nuclear morphometry in prediction of prognosis for rhabdomyosarcoma in children. Histopathology, v. 45, p. 352-9, 2004.

18. MARKOPOULOS, C. et al. Application of the learning vector quantizer to the classification of breast lesions. Anal Quant Cytol Histol, v. 19, p. 453-60, 1997.

19. MEIJER, G.A. et al. Origins of image analysis in clinical pathology. J Clin Pathol, v. 50, p. 365-70, 1997.

20. MILORD, R.A. et al. An objective morphologic parameter to aid in the diagnosis of flat urothelial carcinoma in situ. Human Pathology, v. 32, n. 9, p. 997-1002, 2001.

21. MILLOT, C.; DUFER, J. Clinical applications of image cytometry to human tumor analysis. Histol Histopathol, v. 15 , p. $1185-200,2000$.

22. NATIV, O. et al. The role of nuclear morphometry for predicting disease outcome in patients with localized renal cell carcinoma. Cancer, v. 76, n. 8, p. 1440-4, 1995.

23. ODA, Y.; TSUNEYOSHI M. A comparative study of nuclear morphometry and proliferating activity in neuroectodermal tumors of bone and Ewing's sarcoma of bone. Gen Diag Pathol, v. 141, p. 121-9, 1995.

24. PALCIC, B. Nuclear texture: can it be used as a surrogate endpoint biomarker? J Cell Biochem Suppl, v. 19, p. 40-6, 1994

25. PANTAZOPOULOS, D. Back propagation neural network in the discrimination of benign from malignant lower urinary tract lesions. J Urol, v. 159, p. 1619-23, 1998.

26. POULIN, N. et al. Nuclear morphometry as an intermediate endpoint biomarker in chemoprevention of cervical 
carcinoma using $\alpha$-ifluoromethylornithine. Cytometry (Commun Clin Cytom), v. 38, p. 214-23, 1999.

27. TOSI, P. etal. Nuclear morphometry as an important prognostic factor in stage I renal cell carcinoma. Cancer, v. 58, p. 2512-8, 1986.

28. UTHSCSA ImageTool version 3.0 Final is Here. Disponível em: <http://ddsdx.uthscsa.edu/dig/itdesc.html>. Acesso em: 1 jul. 2007.
29. VELTRI, R.W. et al. Quantitative nuclear grade (QNG): a new image analysis-based biomarker of clinically relevant nuclear structure alterations. J Cell Biochem Suppl, v. 35, p. 151-7, 2000.

30. XU, Y.; PITOT, H. A software package to improve image quality and isolation of objects of interest for quantitative stereology studies of rat hepatocarcinogenesis. Computer Methods and Programs in Biomedicine, n. 81, p. 236-45, 2006. 